\title{
Desempenho cognitivo e motor manual de idosos
}

\author{
Michele Marinho da Silveira ${ }^{1}$ \\ Mirna Wetters Portuguez ${ }^{2}$ \\ ${ }^{1}$ Faculdade Meridional-IMED, RS, Brasil \\ ${ }^{2}$ Pontificia Universidade Católica do Rio Grande do Sul, RS, Brasil
}

\begin{abstract}
Resumo
O desempenho cognitivo e a habilidade motora manual de idosos foram avaliados. A amostra foi constituída por 120 idosos. Os instrumentos utilizados foram: Exame Cognitivo de Addenbrooke Revisado (ACE-R) que avalia domínios como atenção e orientação, memória, fluência, linguagem, habilidades visuo-espaciais; dinamômetro Saehan Corporation ${ }^{\circledR}$ para verificar a força de preensão manual e o painel de habilidades manuais para motricidade fina. Como resultado observou-se que neste estudo a força de preensão manual, a motricidade fina e a cognição dos idosos apresentaram piores desempenhos com o avançar da idade. Houve diferença estatisticamente significativa entre as faixas etárias e os domínios cognitivos memória $(\mathrm{p}=0,022)$, visual-espacial $(p=0,014)$ e total $(p=0,012)$ e associação entre os domínios cognitivos de memória $(p=0,0280)$, fluência $(p=0,003)$, linguagem $(\mathrm{p}<0,001)$, visual-espacial $(\mathrm{p}<0,001)$ e motricidade fina.
\end{abstract}

Palavras-chave: Cognição; Destreza da mão; Avaliação em saúde; Idoso.

\section{Cognitive performance and manual motor of the elderly}

\begin{abstract}
It was evaluated the cognitive performance and the manual motor skill of the elderly. The sample consisted of 120 elderly. The instruments used were: Revised Addenbrooke Cognitive Exam (ACE-R) that evaluates domains such as attention and orientation, memory, fluency, language, visuospatial skills; Saehan Corporation ${ }^{\circledR}$ dynamometer to check the manual grip strength and the fine motor skill panel. As a result, it was observed that in this study, the manual grip strength, the fine motor skills and the cognition of the elderly presented worse performances with advancing age. There was a statistically significant difference between age groups and cognitive domains $(\mathrm{p}=0.022)$, visual-spatial $(\mathrm{p}=0.014)$ and total $(\mathrm{p}=0.012)$ and association between memory cognitive domains $(\mathrm{p}=0.0280),(\mathrm{P}=0.003)$, language $(\mathrm{p}<0.001)$, visual-spatial $(\mathrm{p}<0.001)$ and fine motor skills.
\end{abstract}

Keywords: Cognition; Motor skills; Elderly.

\section{El rendimiento cognitivo y motor de ancianos manual}

\section{Resumen}

Se evaluó el rendimiento cognitivo y la capacidad motora manual de los ancianos. La muestra fue de 120 personas de edad avanzada. Los instrumentos utilizados fueron: El examen cognitivo de Addenbrooke Revisado (ACE-R) que evalúa áreas como la atención y orientación, memoria, fluidez, lenguaje, habilidades visuoespaciales; Saehan Corporación ${ }^{\circledR}$ dinamómetro para verificar panel de la fuerza de prensión y las habilidades manuales para la motricidad fina. Como resultado se observó que esta fuerza estudio del mango, la motricidad fina y la cognición de los adultos mayores tuvieron el peor desempeño con la edad avanzada. Hubo una diferencia estadísticamente significativa entre los grupos de edad y dominios cognitivos de la memoria $(p=0,022)$, visual-espacial $(p=0,014)$ y total $(p=0,012)$ y la asociación entre los dominios cognitivos de la memoria $(p=0,0280)$ fluencia $(p=0,003)$, el lenguaje $(\mathrm{p}<0,001)$, visual-espacial $(\mathrm{p}<0,001)$ y la motricidad fina.

Palabras clave: Cognición; La destreza manual; La evaluación de la salud; Personas de edad avanzada. 


\section{Introdução}

O processo de envelhecimento, aliado ao aumento da expectativa de vida, vem crescendo mundialmente nas últimas décadas. Esse aumento associado ao desenvolvimento de novas estratégias, pesquisas clínicas e ao incremento da investigação sobre questões de saúde pública relacionadas ao envelhecimento, evidenciam uma projeção no número de idosos na sociedade e a sua busca pela melhora da qualidade de vida (Brasil, 2010).

O envelhecimento humano é marcado por mudanças complexas que envolvem os aspectos sensoriais, físicos e cognitivos. As mudanças sensoriais afetam todos os órgãos dos sentidos, ou seja, a visão, a audição, o paladar, o olfato e o tato. As alterações físicas inevitavelmente interferem na coordenação motora, nos reflexos proprioceptivos, na flexibilidade, na força, na velocidade dos movimentos e na resistência muscular (Minúscoli, Meneghini, Oltramari, Wolpat \& Melatti, 2013). Já as cognitivas, podem ocorrer quando idosos se queixam de dificuldades com a memória, atenção, orientação, raciocínio entre outras, particularmente quando comparam o desempenho atual com o do passado (Leite, Winck, Hildebrandt, Kirchner \& Silva, 2012).

Estudos sugerem que em idosos observa-se a diminuição do aprendizado, memória, funções executivas, além de redução do desempenho sensóriomotor, mesmo quando não acometidos por doença (Altermann, Martins, Carpes, \& Mello-Carpes, 2014). Contudo, alterações na função cognitiva parecem ter relação com o desempenho físico do idoso. $\mathrm{O}$ próprio processo de envelhecimento favorece a redução da reserva fisiológica em diferentes sistemas, acarretando diminuição da capacidade de gerar respostas adaptativas frente a estímulos externos. Nesse sentido, contribui para perda progressiva do desempenho físico e alterações do estado cognitivo do idoso, expondo-o, assim, a maior vulnerabilidade (Won et al., 2014; Blankevoor et al., 2013).

Pesquisadores revelam que a motricidade fina, em conjunto com a força muscular, é essencial para o processo do envelhecimento, seja para os membros inferiores presente na marcha, seja para os membros superiores na realização das atividades vitais (Fidelis, Patrizzi, \& Walsh, 2013). A diminuição da motricidade fina e da força muscular, em especial a preensão manual, pode gerar perdas na execução das atividades rotineiras para os idosos, como, por exemplo, abrir um pote de vidro com rosca, cortar um alimento com faca, abotoar a camisa, escovar os dentes, entre outras.
Estudos comprovam a associação entre cognição e habilidade manual (Gallucci et al., 2013; Yassuda et al., 2012), visto que manipular objetos que exijam o controle de força de preensão manual (FPM) agem juntamente com o raciocínio mental, ou seja, não há como o físico e a mente realizarem ações separadas. Por fim, o comprometimento da função física e cognitiva acarreta perda de autonomia e de independência do idoso, sobrecarregando a família, e exigindo maior assistência dos serviços de saúde (Holz, Nunes, Thumé, Lange, \& Facchini, 2013).

Diante do exposto, ressalta-se a necessidade de maior conhecimento acerca das alterações cognitivas do idoso, bem como a influência destas na função física, em especial na força muscular e motricidade fina, contribuindo para a formulação de ações em saúde pública voltadas à prevenção destas condições. Além de nortear intervenções específicas e individuais por parte de profissionais de saúde e procedimentos clínicos envolvidos na reabilitação e manutenção da saúde física e mental dessa população. Desse modo, este estudo buscou avaliar as habilidades cognitivas e motoras manuais de idosos. Como objetivo específico, relacionar essas habilidades com a força de preensão palmar e motricidade fina de idosos.

\section{Método}

\section{Participantes}

Participaram deste estudo transversal e descritivo 120 idosos de um município do norte do Rio Grande do Sul, Brasil e utilizou-se a técnica de amostragem por conveniência. Como critérios de inclusão, considerouse a idade igual ou superior a sessenta anos, fazer parte de grupos de convivência, saber ler e escrever. Além disso, também idosos identificados com transtorno cognitivo leve no teste de Addenbrooke, caracterizados por déficits de memória, mas com preservações de outras funções cognitivas e sem prejuízos em atividades funcionais. Entretanto, foram excluídos, os que apresentaram doenças neurodegenerativas (demências) e doenças ortopédicas nos membros superiores (pós-operatório de cirurgia ortopédica, fratura recente) ou reumatológicas nas mãos (inflamação e deformidades) verificadas por questionários.

\section{Instrumentos}

O questionário sociodemográfico incluiu variáveis, como sexo, idade, estado civil, escolaridade, renda mensal, atividade física, percepção de saúde, patologias, percepção da memória. O Exame Cognitivo 
de Addenbrooke (ACE-R) de Carvalho e Caramelli (2007) é um instrumento breve de rastreio cognitivo, onde a pontuação mais elevada é indicativa do melhor funcionamento cognitivo podendo atingir o máximo de cem pontos e avalia os cinco domínios neurocognitivos: atenção e orientação (18 pontos), memória (26 pontos), fluência (14 pontos), linguagem (26 pontos) e visuo-espacial (16 pontos). Seus pontos de corte são calculados de acordo com a escolaridade: $\geq 50$ pontos para analfabetos, $\geq 60$ pontos para até quatro anos de estudo, $\geq 70$ pontos de cinco a oito anos de estudo, $\geq 80$ pontos mais que nove anos de estudo.

Para avaliar a motricidade fina utilizou-se o painel de habilidades manuais (Andreotti \& Okuma, 1999), formado por um painel retangular $(60 \mathrm{~cm}$ por $15 \mathrm{~cm})$, que contém uma fechadura, uma tomada, um soquete para encaixar a lâmpada e um disco de telefone, dispostos horizontalmente a uma distância de $10 \mathrm{~cm}$ entre cada objeto. Para realizar o teste precisa de um cronômetro, chave, plug e lâmpada. Iniciado o teste, o participante, em pé, em frente ao painel, e com a mão dominante deve colocar a chave na fechadura, o plug na tomada, desencaixar a lâmpada do soquete e discar o número nove no disco do telefone com menor tempo possível. Para isso são feitas duas tentativas, e a de menor tempo será registrada.

Para avaliar a força de preensão manual, o Dinamômetro Saehan Corporation ${ }^{\circledR}$ modelo SH5001 que é um instrumento válido, confiável e comparável com o dinamômetro Jamar ${ }^{\circledR}$ (Reis \& Arantes, 2011). São avaliadas as mãos dominante e não dominante de cada sujeito. O teste foi realizado na posição recomendada pela Sociedade Americana de Terapeutas de Mão: os testados ficaram sentados confortavelmente em uma cadeira sem braços, com os pés apoiados no chão e quadril e joelho posicionados a aproximadamente 90 graus de flexão. O ombro do membro testado ficou aduzido e em rotação neutra, cotovelo em flexão de 90 graus, antebraço na posição neutra e punho entre 0 e 30 graus de extensão e entre 0 a 15 graus de adução. A mão do membro não testado repousou sobre a coxa do mesmo lado. Os participantes são instruídos a manter o posicionamento durante os testes e corrigidos pelo examinador quando necessário. $\mathrm{O}$ protocolo de testes foi dividido em duas partes: na primeira, os testes são realizados inicialmente com a mão dominante (direita) e depois com a mão não dominante (esquerda), de forma não alternada, visto que todos os participantes eram destros. Os participantes são instruídos a fazerem uma contração máxima por três segundos em cada teste. Há um período de descanso de 30 segundos entre cada teste e um período de descanso de 2 minutos entre os testes de cada mão. É utilizada a média dos valores dos três testes de cada mão para a análise dos dados.

\section{Procedimentos}

O estudo foi aprovado pela Comissão Científica do Instituto de Geriatria e Gerontologia e pelo Comitê de Ética em Pesquisa da PUCRS, Porto Alegre, RS, Brasil, sob o parecer $n^{\circ}$ 241.858. Inicialmente, foi realizada uma entrevista individual, cada participante preencheu o termo de consentimento livre e esclarecido e respondeu sobre informações sociodemográficas e de saúde. Na sequência, foram avaliados o desempenho cognitivo (ACE-R) e, após aplicados os testes de MF e FPM. Todos os instrumentos foram aplicados pela autora, para uma padronização dos resultados, visto que os testes aplicados são objetivos e resultam em números. Ao todo foram avaliados 174 indivíduos e foram excluídos do estudo 54 sujeitos.

\section{Análise dos dados}

As variáveis quantitativas foram descritas por média e desvio padrão e as qualitativas por frequências absolutas e relativas. O teste de normalidade Kolmogorov-Smirnov foi aplicado para verificar a distribuição dos dados. Para comparar médias, o teste t-student ou a Análise de Variância (ANOVA) foram aplicados. Para avaliar a correlação entre as variáveis numéricas, o teste da correlação de Pearson foi utilizado. A associação entre as variáveis categóricas foi realizada pelos testes qui-quadrado de Pearson ou exato de Fisher. O nível de significância adotado foi de $5 \%(\mathrm{p}<0,05)$ e as análises foram realizadas no programa SPSS versão 21.0.

\section{Resultados}

Na Tabela 1 estão apresentadas as características sociodemográficas e de percepção de memória e saúde dos idosos participantes. Também são observadas a avaliação da força de preensão palmar e motricidade fina de acordo com a faixa etária e sexo na Tabela 2.

Quando comparadas as faixas etárias na amostra total, quanto à força de preensão manual e motricidade fina, houve diferença significativa apenas na motricidade fina. É possível observar que quanto maior a faixa etária, maior o tempo $(\mathrm{p}<0,001)$. Quando separados por gênero, a mesma associação é observada nas mulheres. No entanto, para os homens, as diferenças entre as faixas etárias foram somente na força de preensão manual não-dominante. Desta forma, quanto maior a faixa etária, menor a força nessa mão. 
TABELA 1

Caracterização da amostra.

\begin{tabular}{|c|c|}
\hline Variáveis & $n=120$ \\
\hline Idade (anos) - média \pm DP & $66,4 \pm 5,8$ \\
\hline \multicolumn{2}{|l|}{ Faixa etária $-\mathrm{n}(\%)$} \\
\hline 60 a 69 anos & $90(75,0)$ \\
\hline 70 a 79 anos & $25(20,8)$ \\
\hline 80 anos ou mais & $5(4,2)$ \\
\hline \multicolumn{2}{|l|}{ Sexo $-n(\%)$} \\
\hline Feminino & $94(78,3)$ \\
\hline Masculino & $26(21,7)$ \\
\hline \multicolumn{2}{|l|}{ Estado Civil - n(\%) } \\
\hline Solteiro (a) & $10(8,3)$ \\
\hline Casado (a) & $57(47,5)$ \\
\hline Separado (a)/Divorciado (a) & $15(12,5)$ \\
\hline Viúvo (a) & $38(31,7)$ \\
\hline \multicolumn{2}{|l|}{ Nível de escolaridade - n(\%) } \\
\hline Até 4 anos & $31(25,8)$ \\
\hline De 5 a 8 anos & $16(13,3)$ \\
\hline De 9 a 11 anos & $40(33,3)$ \\
\hline Acima de 12 anos & $33(27,5)$ \\
\hline \multicolumn{2}{|l|}{ Renda mensal - $\mathrm{n}(\%)$} \\
\hline De 1 a 2 salários mínimos & $100(83,3)$ \\
\hline De 3 a 4 salários mínimos & $17(14,2)$ \\
\hline Acima de 5 salários mínimos & $3(2,5)$ \\
\hline \multicolumn{2}{|l|}{ Percepção de memória - n(\%) } \\
\hline Ótima & $4(3,3)$ \\
\hline Boa & $47(39,2)$ \\
\hline Regular & $57(47,5)$ \\
\hline Ruim & $12(10,0)$ \\
\hline \multicolumn{2}{|c|}{ Atualmente sente-se mais esquecido } \\
\hline Não & $19(15,8)$ \\
\hline Sim & $101(84,2)$ \\
\hline \multicolumn{2}{|l|}{ Percepção de saúde - n(\%) } \\
\hline Ótima & $18(15,0)$ \\
\hline Boa & $57(47,5)$ \\
\hline Regular & $44(36,7)$ \\
\hline Ruim & $1(0,8)$ \\
\hline
\end{tabular}

Quando comparados os gêneros, houve diferença significativa quanto à força de preensão manual dominante na amostra total e na faixa etária de 60 a 69 anos. Quanto à força de preensão manual não dominante, as diferenças entre gêneros ocorreram em praticamente todos os grupos, exceto na faixa de 80 anos ou mais. Para a motricidade fina, a diferença entre os gêneros foi somente no grupo etário de 80 anos ou mais.

Com relação ao desempenho cognitivo dos idosos avaliados pelo ACE-R conforme faixa etária visualiza-se na Tabela 3 que do total da amostra, 22,5\% apresentaram déficit cognitivo conforme o ACE-R. Esse percentual chega a $60 \%$ no grupo de 80 anos ou mais $(p=0,039)$. Quando avaliados os domínios, houve
TABELA 2

Avaliação da força e motricidade fina conforme sexo e faixa etária.

\begin{tabular}{lcccc}
\hline & $\begin{array}{c}\text { Amostra } \\
\text { total } \\
(n=120)\end{array}$ & $\begin{array}{c}\text { Sexo } \\
\text { feminino } \\
(n=94)\end{array}$ & $\begin{array}{c}\text { Sexo } \\
\text { masculino } \\
(n=26)\end{array}$ & $P$ \\
\cline { 2 - 4 } Variáveis & média $\pm D P$ & média $\pm D P$ & média $\pm D P$ & \\
& & & & \\
Força de preensão manual & & & & \\
dominante (kgf) & $24,3 \pm 7,6$ & $21,9 \pm 5,4$ & $32,8 \pm 8,5$ & $<0,001$ \\
$\quad$ Total & $24,5 \pm 7,7$ & $21,9 \pm 5,3$ & $35,4 \pm 7,0$ & $<0,001$ \\
60-69 anos & $24,3 \pm 7,2$ & $22,6 \pm 6,0$ & $28,5 \pm 8,8$ & 0,066 \\
70 -79 anos & $20,4 \pm 9,4$ & $17,0 \pm 5,4$ & $25,5 \pm 14,4$ & 0,394 \\
80 anos ou mais & 0,514 & 0,254 & 0,086 & \\
$P$ & & & & \\
Força de preensão manual & & & & \\
não-dominante (kgf) & $22,7 \pm 7,1$ & $20,3 \pm 5,2$ & $31,3 \pm 6,5$ & $<0,001$ \\
Total & $22,8 \pm 7,2$ & $20,3 \pm 5,1$ & $33,3 \pm 5,2$ & $<0,001$ \\
$60-69$ anos & $23,3 \pm 6,8$ & $21,1 \pm 5,4$ & $29,0 \pm 7,0$ & 0,006 \\
$70-79$ anos & $17,5 \pm 7,3$ & $14,0 \pm 3,5$ & $22,8 \pm 9,7$ & 0,408 \\
80 anos ou mais & 0,252 & 0,092 & 0,047 & \\
$P$ & & & & \\
Motricidade fina (s) & $11,7 \pm 3,4$ & $11,7 \pm 3,5$ & $11,7 \pm 2,9$ & 0,941 \\
Total & $11,0 \pm 3,0$ & $10,9 \pm 2,9$ & $11,8 \pm 3,2$ & 0,226 \\
$60-69$ anos & $13,5 \pm 3,5$ & $14,3 \pm 3,7$ & $11,7 \pm 2,3$ & 0,101 \\
$70-79$ anos & $15,0 \pm 4,4$ & $18,1 \pm 1,1$ & $13,1 \pm 2,5$ & 0,015 \\
80 anos ou mais & $<0,001$ & $<0,001$ & 0,420 & \\
$P$ & & & &
\end{tabular}

diferença significativa entre as faixas etárias quanto a memória $(p=0,022)$, visual-espacial $(p=0,014)$ e total $(\mathrm{p}=0,012)$, sendo que as médias reduzem à medida que aumenta a faixa etária.

Já na Tabela 4 observam-se as correlações entre o desempenho cognitivo dos idosos, força de preensão palmar e motricidade fina havendo uma correlação positiva entre os resultados do domínio Atenção e Orientação com a força de preensão manual dominante $(r=0,186 ; p=0,042)$, sendo que quanto mais o idoso pontua nesse domínio, maior a força na mão dominante. Com a força de preensão manual nãodominante, as correlações não foram significativas $(\mathrm{p}<0,05)$. Em relação à motricidade fina, praticamente todas as correlações inversas foram significativas, exceto para atenção e orientação. Para os demais domínios (memória: $\mathrm{r}=-0,201 ; \mathrm{p}=0,028$; fluência: $r=-0,269 ; p=0,003$; linguagem: $r=-0,341 ; p<0,001$; visual-espacial: $r=-0,366 ; p<0,001$; total: $r=-0,352$; $\mathrm{p}<0,001$ ), quanto maior o tempo, menor o escore nos domínios do ACE-R.

Por fim, os dados de déficit cognitivo não se associaram significativamente com gênero $(p=1,000)$, renda $(p=0,541)$, estado civil $(p=0,058)$, percepção de saúde $(p=0,733)$, percepção de memória $(p=0,555)$. 
TABELA 3

Desempenho cognitivo conforme faixa etária.

\begin{tabular}{lccccc}
\hline Domínios & $\begin{array}{c}\text { Amostra total } \\
(n=120)\end{array}$ & $\begin{array}{c}60-69 \text { anos } \\
(n=90)\end{array}$ & $\begin{array}{c}70-79 \text { anos } \\
(n=25)\end{array}$ & $\begin{array}{c}\text { 80 anos ou mais } \\
(n=5)\end{array}$ & $p$ \\
\cline { 2 - 3 } & média $\pm D P$ & média $\pm D P$ & média $\pm D P$ & média $\pm D P$ & \\
Atenção e orientação & $16,7 \pm 1,8$ & $16,7 \pm 1,7$ & $16,6 \pm 2,1$ & $16,6 \pm 1,9$ & 0,891 \\
Memória & $16,1 \pm 3,6$ & $16,5 \pm 3,5$ & $15,2 \pm 3,9$ & $12,6 \pm 1,5$ & 0,022 \\
Fluência & $9,4 \pm 2,5$ & $9,6 \pm 2,4$ & $9,1 \pm 2,7$ & $7,0 \pm 3,2$ & 0,069 \\
Linguagem & $22,2 \pm 3,4$ & $22,6 \pm 2,9$ & $21,5 \pm 4,4$ & $19,4 \pm 4,7$ & 0,062 \\
Visual-Espacial & $13,4 \pm 2,5$ & $13,7 \pm 2,3$ & $13,0 \pm 3,0$ & $10,6 \pm 2,6$ & 0,014 \\
\hline
\end{tabular}

TABELA 4

Correlação entre o ACE-R, força e motricidade fina por meio do coeficiente de correlação de Pearson (r).

\begin{tabular}{|c|c|c|c|}
\hline Domínios & $\begin{array}{l}\text { Força de preensão } \\
\text { manual dominante }\end{array}$ & $\begin{array}{l}\text { Força de preensão } \\
\text { manual não-dominante }\end{array}$ & Motricidade Fina \\
\hline & Coeficiente de correlação (r) & Coeficiente de correlação $(r)$ & Coeficiente de correlação $(r)$ \\
\hline Atenção e orientação & $0,186^{*}$ & 0,147 & $-0,156$ \\
\hline Memória & $-0,042$ & $-0,080$ & $-0,201^{*}$ \\
\hline Fluência & 0,134 & 0,089 & $-0,269^{* *}$ \\
\hline Linguagem & 0,132 & 0,087 & $-0,341 * * *$ \\
\hline Visual-Espacial & $-0,021$ & $-0,058$ & $-0,366^{* * *}$ \\
\hline Escore Total & 0,085 & 0,032 & $-0,352 * * *$ \\
\hline
\end{tabular}

$* \mathrm{p}<0,05 ; * * \mathrm{p}<0,01 ; * * * \mathrm{p}<0,001$.

\section{Discussão}

Há poucos dados empíricos sobre a relação entre desempenho cognitivo, força de preensão manual e motricidade fina em idosos o que impulsionou a realizar este estudo, pois há uma preocupação atual com o bemestar e melhor qualidade de vida dessas pessoas. Visto que, o processo de envelhecimento da população pode ser acompanhado por meio do declínio das capacidades físicas e cognitivas dos idosos (Argimon \& Stein, 2005).

Com relação à população deste estudo, observou-se que a partir das características sociodemográficas, há uma prevalência do gênero feminino $(78,3 \%)$ enquanto o gênero masculino foi de $21,7 \%$, demonstrando que há uma predominância das mulheres nos grupos de convivência corroborando o estudo de Leite, Winck, Hildebrandt, Kirchner e Silva (2012). A maioria eram idosos casados $47,5 \%$, com predominância de $33,3 \%$ com 9 a 11 anos de estudo, enquanto que $27,5 \%$ possuíam acima de 12 anos e $83,3 \%$ dos idosos recebiam até dois salários mínimos de renda mensal.

A percepção da memória é em grande parte citada como regular $(47,5 \%)$ e boa $(39,2 \%)$, destaca-se a percepção otimista que os idosos têm de si mesmos, em contrapartida ao analisar se sentem mais esquecidos atualmente, $84,2 \%$ revelam que sim e $15,8 \%$ relatam que não. Na própria percepção de saúde, $47,5 \%$ dizem perceber como boa, enquanto que $36,7 \%$ acham regular. Busato, Gallina, Téo, Ferretti e Pozzagnol (2014) revelam que a autopercepção de saúde se apresenta como boa para a maioria dos idosos corroborando esta pesquisa.

No que se refere à avaliação da motricidade fina, tem-se uma queda conforme o passar dos anos, tanto no gênero masculino, como no feminino confirmando os achados de Katzer, Antes e Corazza (2012) e Silveira (2015).

Lindôso, Cammarota, Argimon, Gomes e Schwanke (2011) avaliaram as habilidades manuais de idosos em oficinas de informática, cujos resultados permitiram observar que os idosos se saíram bem no teste com ambos as mãos, porém não apresentaram resultados estatisticamente significativos. Diferentemente deste estudo, que observou que além da motricidade fina diminuir com a idade, ela foi estatisticamente significativa entre as faixas etárias e as mulheres.

Lin et al. (2014) compararam a força de preensão manual e a coordenação bimanual das mãos de jovens e idosos e observaram que o envelhecimento reduz a 
produção de força de preensão manual e aumenta o tempo de desempenho do controle de coordenação das duas mãos deixando-os mais lentos que os jovens, o que pode levar a dificuldades de execução de atividades diárias que exigem ambas as mãos. Os achados desta pesquisa também apresentaram diminuição de FPM e aumento do tempo desempenho da motricidade fina.

Na força de preensão manual, com a mão dominante, houve uma queda de 35,4 para 28,5 e 25,5 para os homens, enquanto que para as mulheres a média fica em 21,9, 22,6 e 17,0, de acordo com as faixas etárias de 60 a 69 anos, 70 a 79 anos e oitenta anos ou mais, essa média de FPM foi similar a outros estudos como o de Virtuoso et al. (2014), Macedo, Freitas e Scheicher (2014) e Sugiura et al. (2013), mas foram diferentes de Viveiro et al. (2014).

Os resultados de FPM demonstram que o nível de força diminui conforme a faixa etária aumenta. Corroborando os resultados encontrados por Ribeiro e Neri (2012) e Reis Filho, Santini, Neves, Fett e Fett (2012). Além disso, observou-se que a FPM é maior em homens que mulheres confirmando os achados de outros estudos (Lenardt et al., 2014; Belmonte et al., 2014).

Com relação aos aspectos cognitivos, observou-se que o declínio cognitivo está de acordo com o aumento da idade, confirmando estes achados com Rodrigues, Ferreira e Haase (2008) e Faria, Lourenço, Ribeiro e Lopes (2013).

Contudo, alterações na função cognitiva parecem ter relação com o desempenho físico do idoso (Won et al., 2014). Um estudo nos Estados Unidos observou que idosos com declínio em funções como atenção e velocidade de processamento também apresentaram prejuízos no desempenho de habilidades como força de preensão e marcha (McGoughet al., 2013). Outro estudo com idosos, na China (Auyeunget al., 2008), observou que déficits de cognição estavam relacionados com FPM ruim, independentemente do ajuste por outras variáveis (idade, massa muscular, presença de morbidades e nível de atividade física).

Além disso, nesta pesquisa houve correlação entre o domínio cognitivo Atenção e Orientação com a força de preensão manual dominante $(r=0,186 ; p=0,042)$, quanto mais o idoso pontuou nesse domínio, maior foi a força (FPM) na mão dominante. Outros estudos reforçam essa correlação de que quanto melhor o desempenho cognitivo melhor a força de preensão manual (Padubidri et al., 2014; Ukegbu, Maselko, Malhotra, Perera, \& Ostby, 2014; Gallucci et al., 2013).

Diante do exposto, a avaliação da função cognitiva e do desempenho físico no idoso, em especial a força muscular, bem como suas possíveis relações, é temática de relevância para as áreas de saúde pública, gerontologia e geriatria, tendo em vista a diminuição da funcionalidade e o aumento da dependência, observados na população idosa. Assim, é possível incentivar o investimento e a alocação de recursos em programas de saúde pública voltados para a integridade da função cognitiva e física no idoso, de modo a favorecer a manutenção ou a melhora de ambas as funções; prevenir ou mesmo retardar a ocorrência de demências e de dependência funcional. Além disso, busca-se incentivar a intersetorialidade na atenção à saúde em geriatria e gerontologia como um eixo estruturador na formulação de estratégias em saúde pública, com base em evidências, de modo a garantir assistência integral no cuidado ao idoso, nos três níveis de atenção à saúde, e favorecer o envelhecimento bem-sucedido.

\section{Referências}

Andreotti, R. A. \& Okuma, S. S. (1999). Validação de uma bateria de testes de atividades devida diária para idosos fisicamente independentes. Revista Paulista de Educação Física, 13(1), 46-66. https://doi.org/10.11606/issn.25945904.rpef.1999.137759

Altermann, C. D. C., Martins, A. S., Carpes, F. P., \& Mello-Carpes, P. B. (2014). Influência da prática mental e observação do movimento sobre a memória motora, função cognitiva e desempenho motor em idosos. Brazilian Journal of Physical Therapy, 18(2), 201-209. https://doi.org/10.1590/S1413-35552012005000150

Argimon, I. L., \& Stein, L. M. (2005). Habilidades cognitivas em indivíduos muito idosos: um estudo longitudinal, Cadernos de saúde Pública, 21(1), 64-72. https://doi.org/10.1590/S0102-311X2005000100008

Auyeung, T. W., Kwok, T., Lee, J., Leung, P. C., Leung, J., \& Woo, J. (2008). Functional decline in cognitive impairment - the relationship between physical and cognitive function. Neuroepidemiology, 31(3), 167-173. https://doi. org/10.1159/000154929

Blankevoort, C. G., Scherder, E., J., Wieling, M. B., Hortobágyi, T., Brouwer, W. H., Geuze, R. H., \&van Heuvelen, M. J. (2013). Physical predictors of cognitive performance in healthy older adults: a cross-sectional analysis. PLoSOne, 8(7). https://doi.org/10.1371/journal.pone.0070799 
Belmonte, L. M., Lima, I. A. X., Belmonte, L. A. O., Gonçalves, V. P., Conrado, J. C., \& Ferreira, D. A. (2014). Força de preensão manual de idosos participantes de grupos de convivência. Revista Brasileira de Promoção de Saúde, 27(1), 85-91. https://doi.org/10.5020/18061230.2014.p85

Brasil. Ministério da Saúde. Secretaria de Atenção à Saúde. Departamento de Ações Programáticas e Estratégicas. (2010). Atenção à saúde da pessoa idosa e envelhecimento. Brasília, DF: Ministério da Saúde.

Busato, M. A., Gallina, L. S., Téo, C. R. P. A., Ferretti, F., \& Pozzagnol, M. (2014). Autopercepção de saúde e vulnerabilidade em idosos. Revista Baiana de Saúde Pública, 38(3), 625-635. https://doi.org/10.5327/Z01000233-2014380300010

Carvalho, V. A., \& Caramelli, P. (2007). Brazilian adaptation of the Addenbrooke's Cognitive ExaminationRevised (ACE-R). Dementia \& Neuropsychologia, 1(2), 212-216. https://doi.org/10.1590/s1980-57642008dn 10200015

Faria, C. A., Lourenço, R. A., Ribeiro, P. C. C., \&Lopes, C. S. (2013). Desempenho cognitivo e fragilidade em idosos clientes de operadora de saúde. Revista de Saúde Pública, 47(5), 923-930. https://doi.org/10.1590/S00348910.2013047004451

Fidelis, L. T., Patrizzi, L. J., \&Walsh, I. A. P. (2013). Influência da prática de exercícios físicos sobre flexibilidade, força muscular e mobilidade funcional em idosos. Revista Brasileira de Geriatria e Gerontologia, 16(1), 109-116. https://doi.org/10.1590/S1809-98232013000100011

Gallucci, M., Mazzuco, S., Ongaro, F., Di Giorgi, E., Mecocci, P., Cesari, M., ... Regini, C. (2013). Bodymass index, lifestyles, physical performance and cognitive decline: the "Treviso Longeva (TRELONG)" study. Journal Nutrition Health Aging, 17(4), 378-384. https://doi.org/10.1007/s12603-012-0397-1

Holz, A. W., Nunes, B. P., Thumé, E., Lange, C., \& Facchini, L. A. (2013). Prevalence of cognitive impairment and associated factors among the elderly in Bagé, Rio Grande do Sul, Brazil. Revista Brasileira de Epidemiologia, 16(4), 880-888. https://doi.org/10.1590/S1415-790X2013000400008

Katzer, J. I., Antes, D. L., \& Corazza, S. T. (2012). Coordenação motora de idosas. Conscientiae Saúde, 11(1), 159-163. https://doi.org/10.5585/conssaude.v11n1.3085

Leite, M. T., Winck, M. T., Hildebrandt, L. M., Kirchner, R. M., \& Silva, L. A. A. (2012). Qualidade de vida e nível cognitivo de pessoas idosas participantes de grupos de convivência. Revista Brasileira de Geriatria e Gerontologia, 15(3), 481-492. https://doi.org/10.1590/S1809-98232012000300009

Lenardt, M. H., Grden, C. R. B., Sousa, J. A. V., Reche, P. M., Betiolli, S. E., \& Ribeiro, D. K. M. N. (2014). Fatores associados à diminuição de força de preensão manual em idosos longevos. Revista da Escola de Enfermagem da USP, 48(6), 1006-1012. https://doi.org/10.1590/S0080-623420140000700007

Lin, C. H., Chou, L. W., Wei, S. H., Lieuc, F. K., Chiangd, S. L., \& Sung, W. H. (2014). Influence of aging on bimanual coordination control. Experimental Gerontology, 53, 40-47. https://doi.org/10.1016/j.exger.2014.02.005

Lindôso, Z. C. L., Cammarota1, M. P., Argimon, I. I. L., Gomes, I., \& Schwanke, C. H. A. (2011). Percepção subjetiva de memória e habilidade manual em idosos de uma oficina de inclusão digital. Revista Brasileira de Geriatria e Gerontologia, 14(2), 303-317. https://doi.org/10.1590/S1809-98232011000200011

Macedo, D. O., Freitas, L. M., \&Scheicher, M. E. (2014). Preensão palmar e mobilidade funcional em idosos com diferentes níveis de atividade física. Fisioterapia e Pesquisa, 21(2), 151-155. https://doi.org/10.1590/18092950/47321022014

McGough, E. L., Cochrane, B. B., Pike, K. C., Logsdon, R. G., McCurry, S. M., \& Teri, L. (2013). Dimensions of physical frailty and cognitive function in older adults with amnestic mild cognitive impairment. Ann Phys RehabilMe, 56(5), 329-341. https://doi.org/10.1016/j.rehab.2013.02.005

Minúscoli, A. V. F., Meneghini, G. O., Oltramari, J. D., Wolpat A., \& Melatti, J. (2013). Síndrome da Fragilidade: elucidando conceitos e propostas de avaliação e tratamento. In C. P. Dias \& J. C. F. Guimarães (Org.). Desvendando o envelhecimento (pp. 45-55). Caxias do Sul, RS: Faculdade da Serra Gaúcha.

Padubidri, A., Snih, S. A., Samper-Ternent, R., Markides, K. S., Ottenbacher, K. J., \& Raji, M. A. (2014). Falls and cognitive decline in Mexican Americans 75 years and older. Clinical Interventions Aging, 9, 719-726. https://doi org/10.2147/CIA.S59448

Reis, M. M., \& Arantes, P. M. (2011). Medida da força de preensão manual - validade e confiabilidade do dinamômetro Saehan. Fisioterapia e Pesquisa, 18(2), 176-181. https://doi.org/10.1590/S1809-29502011000200013

Reis Filho, A. D., Santini, E., Neves, T., Fett, W. C. R., \& Fett, C. A. (2012). Análise do estado nutricional e da força de preensão palmar, lombar e escapular em mulheres de meia idade e idosas. Brazilian Journal of Biomotriciy, 6(4), 245-253.

Ribeiro, L. H. M., \& Neri, A. L. (2012). Exercícios físicos, força muscular e atividades de vida diária em mulheres idosas. Ciências e Saúde Coletiva, 17(8), 2169-2180. https://doi.org/10.1590/S1413-812320120008 00027

Rodrigues, J. L., Ferreira, F. O., \& Haase, V. G. (2008). Perfil do desempenho motor e cognitivo na idade adulta e velhice. Gerais: Revista Interinstitucional de Psicologia, 1(1), 20-33.

Silveira, M. M. (2015). Desempenho cognitivo, estado emocional, qualidade de vida e habilidade motora manual de idosos participantes de oficinas de informática (Tese de Doutorado). Instituto de Geriatria e Gerontologia, Pontifícia Universidade Católica do Rio Grande do Sul, Porto Alegre, RS, Brasil. 
Sugiura, Y., Tanimoto, Y., Watanabe, M., Tsuda, Y., Kimura, M., Kusabiraki, T., \& Kono, K. (2013). Handgrip strength as a predictor of higher-level competence decline among community-dwelling Japanese elderly in an urban area during a 4-year follow-up.Archives of Gerontology and Geriatrics, 57(3), 319-324. https://doi.org/10.1016/j. archger.2013.06.006

Ukegbu, U., Maselko, J., Malhotra, R., Perera, B.,\& Østbye, T. (2014). Correlates of Handgrip Strengt hand activities of daily living in elderly Sri Lankans. Journal of the American Geriatrics Society, 62(9), 1800-1801. https://doi. org/10.1111/jgs.13000

Virtuoso, J. F., Balbé, G. P., Hermes, J. M., Amorim Júnior, E. E., Fortunato, A. R., \& Mazo, G. Z. (2014). Força de preensão manual e aptidões físicas: um estudo preditivo com idosos ativos. Revista Brasileira deGeriatria e Gerontologia, 17(4), 775-784. https://doi.org/10.1590/1809-9823.2014.13183

Viveiro, L. A. P., Almeida, A. S., Meira, D. M., Lavoura, P. H., Carmo, C. M., Silva, J. M., \& Tanaka, C. (2014). Declínio de atividades instrumentais de vida diária associado à perda de força de preensão palmar em idosos internados em enfermaria geriátrica. Revista Brasileira de Geriatria e Gerontologia, 17(2), 235-242. https://doi.org/10.1590/ S1809-98232014000200002

Won, H., Singh, D. K., Din, N. C., Badrasawi, M., Manaf, Z.A., Tan, S.T., ... Shahar, S. (2014). Relationship between physical performance and cognitive performance measures among community-dwelling older adults. Journal of Clinical Epidemiology, 6, 343-350. https://doi.org/10.2147/CLEP.S62392

Yassuda, M. S., Lopes, A., Cachioni, M., Falcao, D. V., Batistoni, S. S., Guimaraes, V. V., \& Neri, A. L. (2012).Frailty criteria and cognitive performance are related: data from the FIBRA study in Ermelino Matarazzo, São Paulo, Brazil. Journal Nutrition Health Aging, 16(1), 55-61. https://doi.org/10.1007/s12603-012-0003-6

Dados dos autores:

Michele Marinho da Silveira - Pós-Doutora, Faculdade Meridional - IMED

Mirna Wetters Portuguez - Doutora, Pontifícia Universidade Católica do Rio Grande do Sul.

Endereço para correspondência:

Michele Marinho da Silveira

Rua Carlos Gomes, 336/1004

99070-060 Passo Fundo, RS, Brasil

$<$ michele.msilveira@gmail.com>

Recebido em: 19.03.2017

Aceito em: 05.07.2017 